\title{
An empirical approach combining nuclear physics and dense nucleonic matter
}

\section{Jérôme Margueron*}

Univ Lyon, Université Lyon 1, IN2P3-CNRS, Institut de Physique Nucléaire de Lyon, F-69622 Villeurbanne, France

E-mail: j.margueron@ipnl.in2p3.fr

\section{Rudiney Casali}

Departamento de Fśica, Instituto Tecnológico de Aeronáutica, CTA, Sã̃ José dos Campos, S.P., Brazil

The nuclear equation of state is still a very challenging issue for nuclear astrophysics, for the determination of masses and radii of neutron stars as well as properties of core-collapse supernovae. We aim in this work at understanding the important nuclear physics properties which influence the nuclear equation of state based a flexible approach where the nuclear properties are encoded in the empirical parameters. We also checked that this phenomenological equation of state is able to mimic most of existing modelings. We first apply this approach to the understanding of the relation between masses/radii of neutron stars and the nuclear properties, and we show that the most determinant ones are $L_{s y m}$ and $K_{\text {sym }}$ acting in the isovector channel.

The Modern Physics of Compact Stars 2015

30 September 2015 - 3 October 2015

Yerevan, Armenia

${ }^{*}$ Speaker. 


\section{Nuclear Matter Properties: The Present Uncertainties}

In their seminal study of neutron stars hydrostatic equations, Tolman, Oppenheimer and Volkoff have shown that without nuclear interaction neutron stars would not exceed about $0.7 \mathbf{M}_{\odot}[1,2]$, while the canonical neutron stars mass is about $1.45 \mathrm{M}_{\odot}$ [3], and recent observation analysis have reported the existence of about $2 \mathrm{M}_{\odot}$ neutron stars $[4,5]$. The nuclear interaction therefore plays a crucial role in the determination of the mass of neutron stars, as well as of their radius. At present time, measures of neutron stars radii are still quite uncertain but recent observational progress are expected to provide more stringent bounds [6].

The uncertainty in the masses and radii of neutron stars could be related to the uncertainty in the empirical parameters of nuclear matter. These empirical properties are determined from nuclear physics experiments such as nuclear masses measurements, determination of charge-density profiles, excitation of collective modes (ISGMR, IVGDR, etc...), e.g. see Refs. [7, 8, 9] and references therein. Expressing the binding energy in terms of isoscalar and isovector energies, with $n=n_{n}+n_{p}$ and $\delta=\left(n_{n}-n_{p}\right) / n$,

$$
e(n, \delta)=e_{I S}(n)+\delta^{2} e_{I V}(n)
$$

the empirical parameters are identified as the coefficients of the following power expansion,

$$
\begin{aligned}
& e_{I S}(n)=E_{\text {sat }}+\frac{K_{\text {sat }}}{2} x(n)^{2}+\frac{Q_{\text {sat }}}{6} x(n)^{3}+\ldots, \\
& e_{I V}(n)=E_{\text {sym }}+L_{\text {sym }} x(n)+\frac{K_{\text {sym }}}{2} x(n)^{2}+\frac{Q_{s y m}}{6} x(n)^{3}+\ldots,
\end{aligned}
$$

where $x(n)=\left(n-n_{\text {sat }}\right) /\left(3 n_{\text {sat }}\right), n_{\text {sat }}$ being the saturation density of symmetric nuclear matter. For a clarification of the various definitions of empirical parameters existing in the literature, we refer to the very clear discussion in the appendix of Ref. [10].

\begin{tabular}{ccccccccccccc}
\hline & & $\begin{array}{c}E_{\text {sat }} \\
\text { Medel }\end{array}$ & $\begin{array}{c}E_{\text {sym }} \\
\mathrm{MeV}\end{array}$ & $\begin{array}{c}n_{\text {sat }} \\
\mathrm{Mm}^{-3}\end{array}$ & $\begin{array}{c}L_{\text {sym }} \\
\mathrm{MeV}\end{array}$ & $\begin{array}{c}K_{\text {sat }} \\
\mathrm{MeV}\end{array}$ & $\begin{array}{c}K_{\text {sym }} \\
\mathrm{MeV}\end{array}$ & $\begin{array}{c}Q_{\text {sat }} \\
\mathrm{MeV}\end{array}$ & $\begin{array}{c}Q_{\text {sym }} \\
\mathrm{MeV}\end{array}$ & $\begin{array}{c}k_{\tau} \\
\mathrm{MeV}\end{array}$ & $m_{s}^{*} / m$ & $\Delta m^{*} / m$ \\
$\left(N_{\text {model }}\right)$ & der. order & 0 & 0 & 1 & 1 & 2 & 2 & 3 & 3 & - & - & - \\
\hline Skyrme & Average & -15.82 & 30.14 & 0.158 & 52.0 & 242.3 & -127.9 & -326.4 & 292.4 & -362.7 & 0.8 & 0.2 \\
$(16)$ & $\sigma$ & 0.16 & 1.71 & 0.004 & 23.6 & 32.3 & 104.9 & 113.9 & 156.7 & 42.9 & 0.1 & 0.3 \\
\hline RMF & Average & -16.24 & 35.11 & 0.149 & 90.2 & 268.0 & -4.6 & -1.9 & 271.1 & -548.8 & 0.67 & -0.08 \\
$(11)$ & $\sigma$ & 0.06 & 2.63 & 0.003 & 29.6 & 33.5 & 87.7 & 392.5 & 357.1 & 153.1 & 0.02 & 0.03 \\
\hline RHF & Average & -15.97 & 33.97 & 0.154 & 90.0 & 248.1 & 128.2 & 389.2 & 523.3 & -571.8 & 0.74 & -0.02 \\
$(4)$ & $\sigma$ & 0.08 & 1.37 & 0.004 & 11.1 & 11.6 & 51.1 & 350.4 & 236.8 & 169.1 & 0.03 & 0.00 \\
\hline Total & Average & -16.03 & 33.41 & 0.154 & 78.7 & 251.6 & 4.6 & 13.8 & 376.7 & -494.3 & 0.72 & 0.00 \\
$(31)$ & $\sigma$ & 0.20 & 2.70 & 0.005 & 30.3 & 28.1 & 132.5 & 430.0 & 297.2 & 164.6 & 0.09 & 0.20 \\
\hline
\end{tabular}

Table 1: The average empirical parameters and the standard deviation calculated for the different models (Skyrme, RMF, RHF) are shown. The standard deviation is defined as $\sigma=\sqrt{\sum_{i} \omega_{i}\left(x_{i}-\langle x\rangle\right)^{2}}$, where $\omega_{i}$ is the weight which is defined such that the influence of difference types of model is equally distributed $\left(\omega_{i}=1 /\left(3 N_{\text {model }}\right)\right.$. 
Some of these empirical parameters are quite well constrained by nuclear properties, e.g. $E_{s a t}$, $n_{\text {sat }}, K_{\text {sat }}$ and $E_{\text {sym }}$, while the others are yet quite uncertain. In order to determine a realistic range of variation for the uncertain empirical parameters, we have compared the predictions provided by various kinds of nuclear interactions. We have selected a set of Skyrme (non-relativistic) and RMF and RHF (relativistic) models for which the values of the empirical parameters have been calculated [11]. In addition to the empirical parameters, we have calculated the value of the parameter $k_{\tau}=K_{\text {sym }}-6 L_{\text {sym }}-\frac{Q_{\text {sat }}}{K_{\text {sat }}} L_{s y m}$. and the effective mass in symmetric matter $\frac{m_{s}^{*}}{m}$ as well as the isospin splitting in neutron matter $\frac{\Delta m^{*}}{m}$. In this set, we have selected 16 Skyrme (non-relativistic) type models over several tenth (by taking only one model per group producing these models), 11 RMF and 4 RHF (relativistic) models [11]. To better discriminate between well known parameters and the unknown ones, we have calculate the average values and the standard deviations for the empirical parameters for each of the three kinds of nuclear models. The results are shown in Table 1. In addition, since some of these parameters strongly depend of the kind of nuclear models, the global average and standard deviations for all models have also been calculated. More details concerning the interactions considered in our selection will be given in a forthcoming publication [11]. It is clear from Table 1 that some empirical quantities, e.g. $E_{s a t}, E_{s y m}, n_{s a t}$ and $K_{\text {sat }}$, are better determined than others, e.g. $L_{\text {sym }}, K_{\text {sym }}, Q_{\text {sat }}$ and $Q_{\text {sym }}$.

It is interesting to notice that the standard deviation obtained for the incompressibility $K_{\text {sat }}$ and the slope of the symmetry energy $L_{\text {sym }}$ are compatible with the present experimental uncertainties [7, 8, 9]. It can also be remarked that parameters like $Q_{\text {sat }}$ or $K_{\text {sym }}$ are very much model dependent. For instance the sign of $Q_{\text {sat }}$ and $K_{s y m}$ is opposite between Skyrme and RHF models, while RMF models predict for these two empirical parameters values compatible with 0 . The value of $k_{\tau}$ is surprisingly rather stable are quite model independent, due to compensation between the uncertainties of $L_{\text {sym }}, K_{\text {sym }}$ and $Q_{\text {sat }}$.

\section{The Empirical Equation of State}

In this section, we investigate to which extend a series expansion of the same kind as the one given by Eqs. (1.2)-(1.3) could generate an empirical equation of state (EOS). There are two questions to answer, which are i) does the density and isospin dependence is rich enough in the series expansion, and ii) what is the convergence radius in density and isospin parameter of such series expansions.

The model on which the empirical EOS is based on only four requirements:

1. We consider nucleons as non-relativistic particles.

2. The nuclear potential is quadratic in the isospin asymmetry parameter $\delta$.

3. The EOS is analytic in the parameter $x$, which justify the series expansion in this parameter.

4. The binding energy satisfies the following $\operatorname{limit}: \lim _{n_{0} \rightarrow 0} e\left(n_{0}, n_{1}\right)=0$.

To do so, we have extended the simple model (1.1) by separating the contribution of the kinetic energy from the one of the potential energy, and added a low density correction to get the correct 


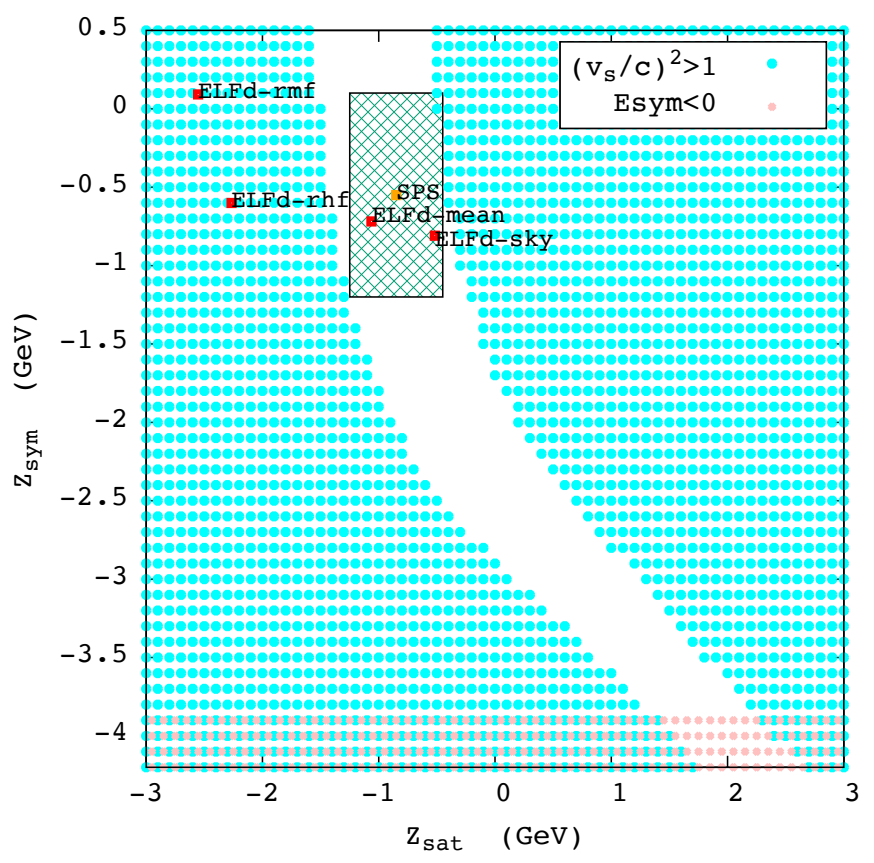

Figure 1: Allowed region of variation for the empirical parameters $Z_{s a t}$ and $Z_{s y m}$ where the regions excluded by acausality and negative symmetry energy are shown in blue and red points.

limit at $n=0$,

$$
e^{N}(n, \delta)=t(n, \delta)+\sum_{\alpha=0}^{N} \frac{v_{\alpha}(\boldsymbol{\delta})}{\alpha !} x(n)^{\alpha} u_{\alpha}^{N}(n),
$$

where $t(n, \delta)$ is the energy of a free Fermi gas (eventually corrected by an in-medium effective mass), and the function $u_{\alpha}^{N}(n)=1-(-3)^{N+1-\alpha} \exp \left(-b n / n_{s a t}\right)$. See Ref. [11] for more details in the expression (2.1). There is still a one to one correspondance between the parameters of the model, $v_{\alpha}(\boldsymbol{\delta})$, and the empirical parameters of nuclear matter.

We have checked that the empirical energy per particle (2.1) could very accurately reproduce a large set of known equation of state [11].

In the following we explore the possible values for the empirical parameters $Z_{\text {sat } / \text { sym }}, Q_{\text {sat } / \text { sym }}$, $K_{\text {sat } / \text { sym }}$, and $L_{\text {sym }}$ and $E_{\text {sym }}$, considering a large domain of variation as suggested by Tab. 1 and excluding parameters which lead to supra-luminosity and $S\left(n_{0}\right)<0$ in the density range $\left[n_{\text {sat }}, 4 n_{\text {sat }}\right]$. The proton fraction that we considered hereafter is the one which satisfy $\beta$-equilibrium.

We give here an illustration of the constraints previously presented in the case of the parameters $Z_{\text {sat } / \text { sym }}$. The parameters $Z_{\text {sat } / \text { sym }}$ are varied from -3000 to $3000 \mathrm{MeV}\left(Z_{\text {sat }}\right)$ and from -4500 up to $500 \mathrm{MeV}\left(Z_{\text {sym }}\right)$ in Fig. 1 . The region for the parameters $Z_{\text {sat } / \text { sym }}$ where the EoS is acausal for densities ranging from $n_{\text {sat }}$ to $4 n_{\text {sat }}$ is shown by the region filled with blue symbols. The region for which the symmetry energy becomes negative is is represented with the pink symbols. Fig. 1 show that there is a very small region for the parameters $Z_{\text {sat } / \text { sym }}$ which satisfy both causality and positiveness of the symmetry energy. Are also shown in Fig. 1 the points corresponding to the 


\begin{tabular}{|c|c|c|c|c|c|c|c|c|c|c|c|c|}
\hline & $E_{s a t}$ & $E_{s y m}$ & $n_{s a t}$ & $L_{s y m}$ & $K_{s a t}$ & $K_{\text {sym }}$ & $Q_{\text {sat }}$ & $Q_{s y m}$ & $Z_{\text {sat }}$ & $Z_{\text {sym }}$ & $m_{\text {sat }}^{*} /$ & ${ }^{*}{ }_{s a t} / m$ \\
\hline & $\mathrm{MeV}$ & $\mathrm{MeV}$ & $\mathrm{fm}^{-3}$ & $\mathrm{MeV}$ & $\mathrm{MeV}$ & $\mathrm{MeV}$ & $\mathrm{MeV}$ & $\mathrm{MeV}$ & $\mathrm{MeV}$ & $\mathrm{MeV}$ & & \\
\hline SP & 16.0 & 33.0 & 0. & 70 & 240 & 30 & 35 & 120 & -950 & -550 & 0.7 & 0 . \\
\hline$\sigma$ & \pm 0.5 & \pm 3 & \pm 0.005 & \pm 40 & \pm 40 & \pm 170 & \pm 235 & \pm 320 & \pm 550 & \pm 950 & \pm 0.1 & \pm 0.1 \\
\hline
\end{tabular}

Table 2: Standard parameter set (SPS) and variation (identified as $\sigma$ ).

average values obtained from various models, more details are provided in Ref. [11]: for Skyrme (ELFd-sky), RMF (ELFd-rmf), RHF (ELFd-rhf), and for the average over all models (ELFd-mean). These points are marked in red. Notice that the average values ELFd-rmf and ELFd-rhf are outside the authorized region. This might be because we are considering a non-relativistic framework for our analysis while these points correspond to relativistic approaches. We aim in the future at extending our approach to a relativistic one to better understand this point. For the present work, we define a standard parameter set (SPS) considering mainly non-relativistic models. We have considered the largest square around the average point ELFd-mean shown as a filled light-blue box in the figure, and we have decided to take for the SPS definition the center of the box identified with the orange point. The values and their variation for the parameters $Z_{\text {sat } / \text { sym }}$ are given in Tab. 2 .

More details are provided in Ref. [11] where similar figures are performed for $Q_{\text {sat } / \text { sym }}, K_{\text {sat } / \text { sym }}$, and $L_{s y m}$ and $E_{s y m}$. A summary of the empirical parameters which satisfy the causality and the symmetry energy conditions is presented in Tab. 2. In the following, the summary presented in Tab. 2 defines a standard parameter set (SPS) and the variation around this SPS which still satisfy these conditions.

\section{MR Diagram}

Considering the SPS and its uncertainties provided in Tab. 2, we now analyse how much the MR diagram is impacted by changing the value of the empirical parameters, and which empirical parameters are the more crucial ones for the determination of masses and radii of neutron stars. To do so, we solve the hydrostatic equations in general relativity for spherical and non-rotating stars, also named the TOV equations $[1,2]$.

We first represent in Fig. 2 the impact of the IS empirical parameters $K_{s a t}$ and $Q_{\text {sat }}$ (the impact of $E_{s a t}$ and $n_{s a t}$ being extremely weak) on the mass-radius relationship. $K_{s a t}$ and $Q_{s a t}$ are varied within the SPS defined in Tab. 2. The pink-colored region in Fig. 2 stands for the observed masses, e.g. between $1.2 \mathrm{M}_{\odot}$ and $2.0 \mathrm{M}_{\odot}$. The crosses represent the value of the central density in units of $n_{\text {sat }}$.

Varying $K_{\text {sat }}$, the impact on the radius at the canonical mass is about $300 \mathrm{~m}(1.3 \mathrm{~km}$ at the maximal mass). A similar impact if found for $Q_{\text {sat }}$ while it can be observed that the negative boundary for $Q_{s a t}$ leads to a too-soft EOS which is stopped when $\left(v_{s} / c\right)^{2}<0$. This is the reason why the mass-radius curve is stopped just above $4 n_{\text {sat }}$ for this case. It can also be observed that as the value of the empirical parameter $K_{s a t}$ or $Q_{\text {sat }}$ increases, the EOS becomes more stiff, and the NS radius increases.

We now discuss the impact of the IV empirical parameters $E_{s y m}, L_{s y m}, K_{s y m}$ and $Q_{\text {sym }}$ on the mass-radius relationship in Fig. 3. The impact of $E_{\text {sym }}$ is small, as expected, while it is clear that 

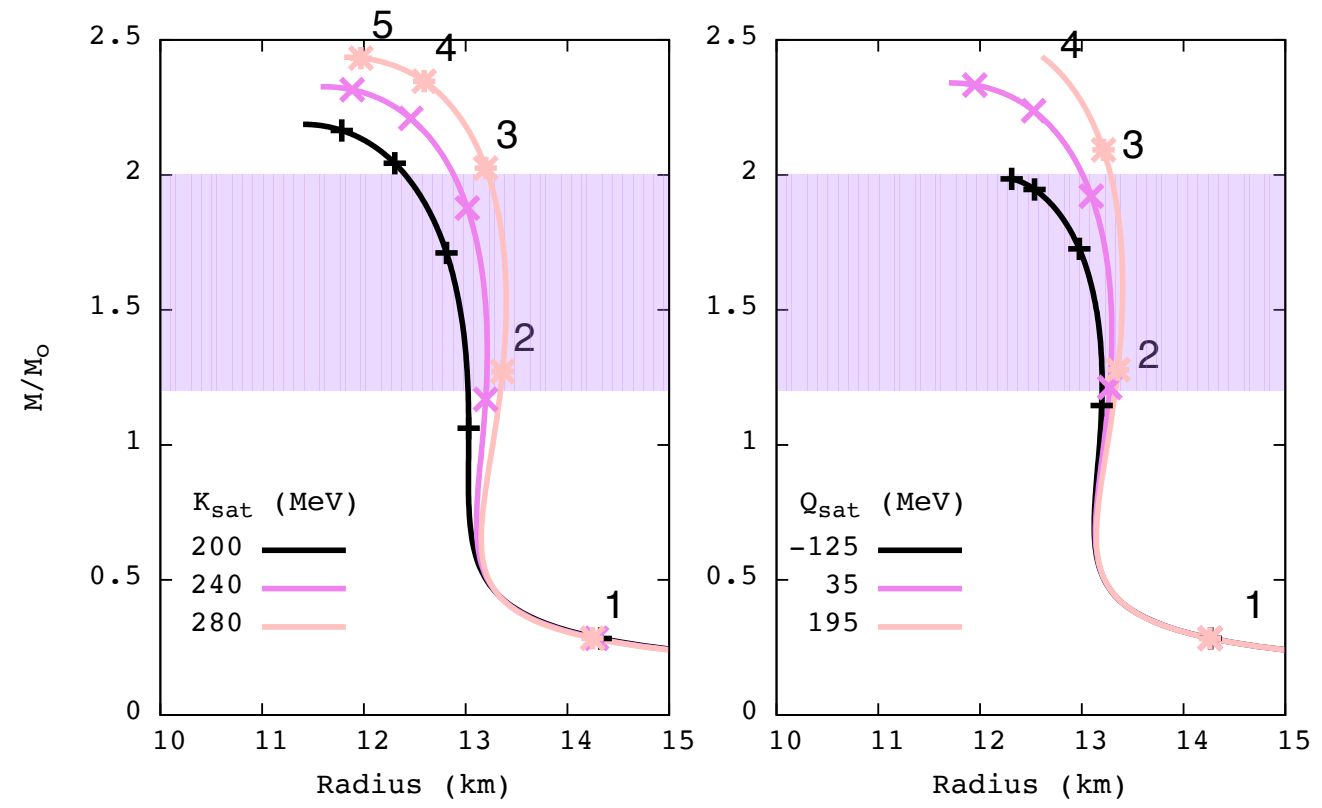

Figure 2: Impact of various isoscalar empirical parameters $\left(K_{s a t}, Q_{s a t}\right)$ on the mass/radius diagram.

both $L_{s y m}$ and $K_{\text {sym }}$ have a very important impact. The effect of $L_{s y m}$ leads to an uncertainty of about $2 \mathrm{~km}$ at NS canonical masses and of about $1 \mathrm{~km}$ at $2 \mathrm{M}_{\text {odot }}$. The effect of $K_{\text {sym }}$ is also quite large for low mass NS (about $1.5 \mathrm{~km}$ ), but the lowest value of $K_{\text {sym }}$ that we have considered gives a too soft EOS which is stopped at around $3 n_{\text {sat }}$. it is interesting to relate the domain in mass and radii where $L_{\text {sym }}$ and $K_{\text {sym }}$ have their largest impact, with the central density. We observe that the impact of $L_{\text {sym }}$ is observed just after $n_{\text {sat }}$ and for NS masses of about $0.5 M_{\odot}$, while the impact of $K_{\text {sym }}$ comes for slightly larger densities, above $1.5 n_{\text {sat }}$ and therefore slightly larger NS masses.

The impact of $Q_{s y m}$ is smaller since the empirical parameter modifies the EOS at high density and its values authorized by causality and symmetry energy positiveness conditions does not vary in a very large range.

In summary, we have shown that the largest impact on the mass-radius relationship is provided by the following empirical parameters: $L_{s y m}$ and $K_{s y m}$. It is expected that $L_{s y m}$ may be determined from accurate measurement of the neutron skin for ${ }^{208} \mathrm{~Pb}$ [12], while $K_{\text {sym }}$ is more difficult to determine. More may hope that systematical analysis of the isoscalar giant monopole resonance may shed some light on a better determination of this parameter. From neutron star observations, our analysis show that an interesting contraint will be the measurement of a radius of a low mass neutron star. We are still far from accurate radius measurement of such objects, but this is the aim of future $\mathrm{x}$-ray observatories as for instance NICER.

\section{Conclusions}

We have presented an empirical model for the nuclear equation of state which can reproduce most of the known equation of states, provided the parameters are well chosen. This EOS is based on nucleonic degrees of freedom and assumes matter in non-relativistic regime. It allows a natural 

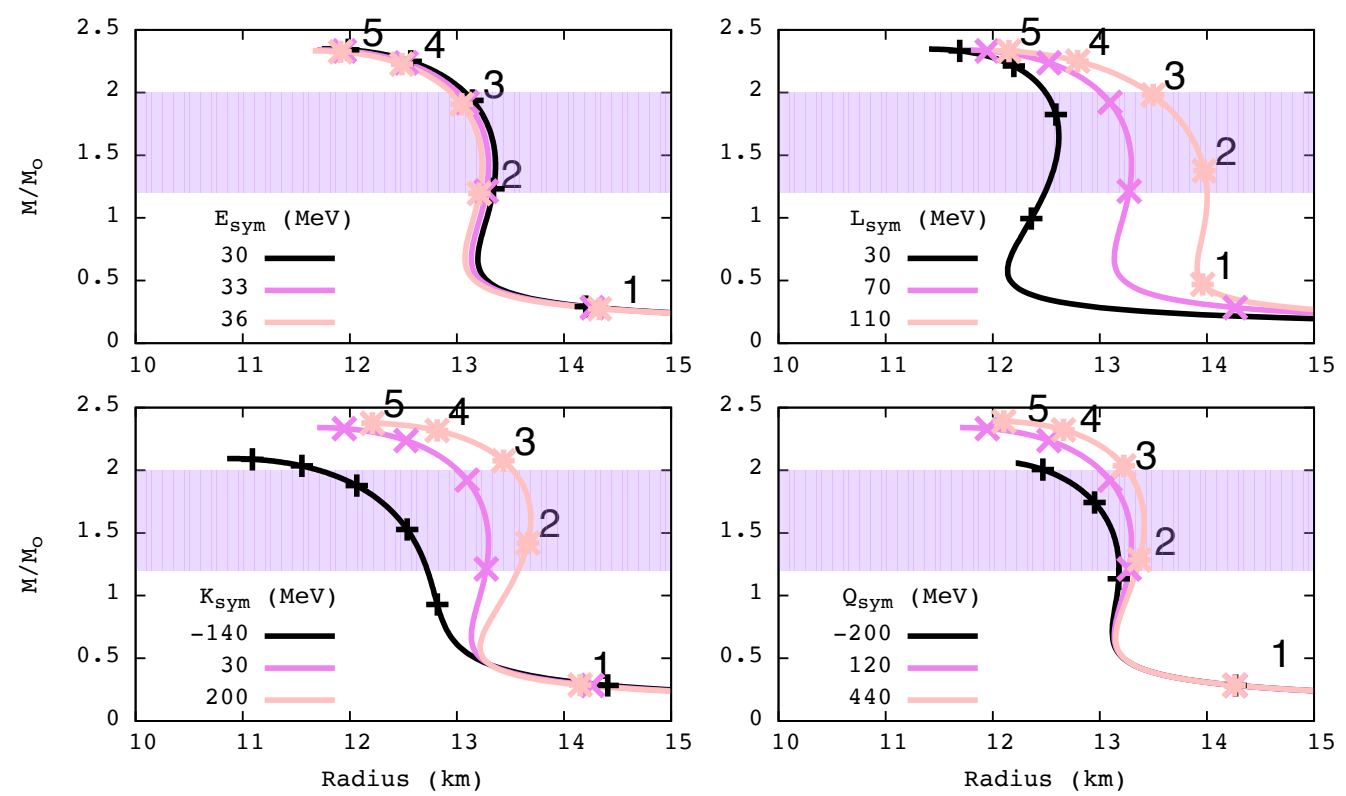

Figure 3: Impact of various isovector empirical parameters $\left(E_{s y m}, L_{s y m}, K_{s y m}, Q_{s y m}\right)$ on the mass/radius diagram.

implementation of our best knowledge on the nuclear empirical parameters which is based on nuclear experiments on Earth.

Since this EOS is controlled by the nuclear empirical parameters, we have analyzed the range of variation for these parameters, first based on a statistical analysis of 50 microscopical models, and then better constrained by two conditions in $\beta$-equilibrated matter: causality and positiveness of the symmetry energy. This have conducted us to the definition of a standard parameter set (SPS) with both average and variation for each of the empirical parameters. This model has been used to understand the relation between the mass and radius of neutron stars and the empirical parameters. We have deduced that the main source of uncertainties on the dense matter EOS is coming from the uncertainties in the empirical parameters $L_{\text {sym }}$ and $K_{\text {sym }}$.

In the future, the empirical equation of state presented here could be applied to other situations, such as for instance the study of several phenomenons related to neutron star evolution ( $\mathrm{x}$-ray emission for instance), as well as core collapse supernovae.

\section{References}

[1] R.C. Tolman, Phys. Rev. 55, 364 (1939).

[2] J.R. Oppenheimer, \& G.M. Volkoff, Phys. Rev. 55, 379 (1939).

[3] J.M. Lattimer \& M. Prakash, Phys. Rev. Lett. 94, 111101 (2005).

[4] Demorest, et al., Nature, 467(7319), 1081 ?1083 (2010).

[5] J. Antoniadis, et al., Science 340, 1233232 (2013).

[6] A.W. Steiner, J.M. Lattimer, \& E.F. Brown, Astro. J. 765, L5 (2013). 
[7] M.B. Tsang et al., Phys. Rev. C 86, 015803 (2012).

[8] X. Roca-Maza et al., Phys. Rev. C 87, 034301 (2013).

[9] E. Khan, J. Margueron \& I. Vidaña, Phys. Rev. Lett. 109, 092501 (2012); E. Khan \& J. Margueron, Phys. Rev. C 88, 034319 (2013).

[10] J. Piekarewicz and M. Centelles, Phys. Rev. C 79, 054311 (2009).

[11] J. Margueron, R. Casali, and F. Gulminelli, in preparation.

[12] C. J. Horowitz, J. Piekarewicz, Phys. Rev. Lett. 86, 5647 (2001). 